\title{
Evolución cosmológica de un modelo de energía oscura con interacción no lineal
}

\author{
Cosmological Evolution of a Dark Energy Model with Non-Linear Interaction
}

A. Oliveros ${ }^{\mathrm{a}, *}$

Recepción: 13-oct-2015

Aceptación: 20-dic-2015

\begin{abstract}
Resumen
En este trabajo consideramos un modelo holográfico de energía oscura junto con una interacción no lineal entre la componente de energía oscura y la de materia oscura en un universo plano tipo FRW. En este contexto, se analiza la evolución cosmológica del universo teniendo en cuenta el comportamiento de los parámetros de desaceleración $q$ y de la ecuación de estado $w_{\Lambda}$. A partir de este análisis se deduce que el universo presenta una fase de expansión acelerada en tiempos tardíos. Se observa también que en este escenario la densidad de energía oscura presenta una singularidad tipo Big Rip en el futuro. Finalmente, comparamos el modelo con los datos disponibles actualmente para $H(z)$.
\end{abstract}

Palabras clave: cosmología, energía oscura.

\begin{abstract}
In this paper it is considered a holographic dark energy model with a non-linear interaction between the component of dark energy and dark matter in a FRW type flat universe. In this context, the cosmological evolution of the universe is analyzed taking into account the behavior of the deceleration parameters $q$ and the equation of state $w_{\Lambda}$. From this analysis it follows that the universe has a phase of accelerated expansion in late times. It also notes that in this scenario the dark energy density will have a Big Rip type singularity in the future. Finally, it is compared the model with currently available data for $H(z)$.
\end{abstract}

Key words: Cosmology, Dark Energy.

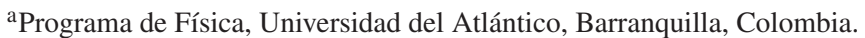

*Autor de correspondencia: alexanderoliveros@mail.uniatlantico.edu.co
} 


\section{Introducción}

La existencia de la energía oscura fue postulada por varios grupos de investigación en 1998 para poder explicar el régimen de expansión acelerada del universo actual, el cual se constituye en uno de los grandes misterios sin resolver en la física, ya que su origen, desde el punto de vista fundamental, es aún desconocido. Este regimen de expansión acelerada se descubrió a partir de la gran cantidad de información astrofísica obtenida al realizar las medidas de luminosidad en las supernovas tipo Ia (SNIa) [1], así como al estudiar las anisotropías observadas en la radiación cósmica de fondo (CMB) [6] y análizar la estructura a gran escala del universo (LSS) [8]. La propuesta más simple para representar la energía oscura es la constante cosmológica ([11], [12], [13]), pero tiene dos problemas, como lo son el problema del ajuste y el de la coincidencia. Se han considerado en la literatura gran variedad de modelos con campos escalares como alternativas diferentes para explicar la naturaleza de la energía oscura, entre los que se tienen los campos de quintaesencia, k-esencia, taquión y el dilatón [14]. Otras propuestas de explicación de la naturaleza de la energía oscura, incluyen modelos de mundos brana ([15], [13]); teorías modificadas de la gravitación (gravitación $F(R), F(G), F(R, G)$ ), en las cuales el régimen de expansión acelerada surge de una modificación de la geometría [16], sin necesidad de invocar una componente de energía oscura. Modelos de energía oscura con ecuaciones de estado poco usuales ([17], [18]) también han sido considerados. Otra aproximación muy interesante, y ampliamente estudiada, al problema de la energía oscura es un modelo inspirado en la gravitación cuántica, conocido como el principio holográfico (para más detalles sobre esta propuesta ver [19]). En este trabajo vamos a considerar la propuesta introducida por Granda y Oliveros en [20], donde se considera que la densidad de energía oscura holográfica depende del término cuadrático usual en el parámetro de Hubble $(H)$ y también de la derivada en el tiempo de $H$, es decir, $\rho_{\Lambda}=3 M_{p}^{2}\left(\alpha H^{2}+\beta \dot{H}\right)$. Este modelo ha sido ampliamente estudiado en la literatura en varios contextos y se le conoce como nuevo modelo holográfico de energía oscura (NHDE). Dentro de la gran variedad de escenarios propuestos para abordar el problema de la energía oscura, también se considera la posibilidad de introducir un término $Q$ de interacción entre las componentes de energía oscura y de materia oscura. Recientemente, en [21] se muestra a partir de las observaciones cosmológicas que es posible tener una interacción entre energía oscura y de materia oscura. Usualmente se introduce el término de interacción $Q$ con el propósito de evitar el problema de la coincidencia (en [22] se encuentra una revisión reciente sobre este tópico). Debido a que no se conoce el origen fundamental de la energía oscura, el término de interacción se introduce bajo consideraciones fenomenológicas. Usualmente, el término de interacción es proporcional a la densidad de energía oscura, la materia oscura o una combinación lineal de ellas (ver [23] y las referencias allí citadas). El signo en el término de interacción $Q$ determina la dirección del flujo de energía. Si $Q$ es positivo, el decaimiento es de energía oscura en materia oscura y si $Q$ es negativo, el decaimiento es de materia oscura en energía oscura. También, algunos autores han considerado la posibilidad de tener términos de interacción no lineales ([23], [24], [25], [26], [27], [28]). En este trabajo vamos a considerar esta última posibilidad, para un caso concreto en la escogencia del término de interacción $Q$, con el cual podemos obtener soluciones analíticas y fenomenológicamente viables.

Este artículo está organizado del siguiente modo: en la sección 2 introduciremos el modelo holográfico de energía oscura, junto con el término de interacción no lineal entre energía oscura y materia oscura, y se obtienen las cantidades básicas necesarias para los cálculos posteriores. En la sección 3 se analiza la evolución cosmológica del modelo. Finalmente, en la sección 4 se presentan algunas conclusiones.

\section{El modelo}

Las ecuaciones diferenciales que determinan el factor de escala $a(t)$ y la densidad de materia $\rho$ se obtienen a partir de las ecuaciones de Einstein

$$
R_{\mu \nu}-\frac{1}{2} R g_{\mu v}=8 \pi G T_{\mu \nu}
$$

donde $R_{\mu v}$ es el tensor de Ricci (el cual depende de la métrica $g_{\mu \nu}$ y de sus derivadas), $R$ es el escalar de Ricci y $T_{\mu \nu}$ es el tensor de energía-impulso, el cual describe el contenido material del universo.

La escogencia más general de la métrica que satisface las condiciones de homogeneidad e isotropía del universo a gran escala es la métrica de Friedmann, 
Robertson-Walker (FRW)

$$
d s^{2}=-d t^{2}+a^{2}(t)\left[\frac{d r^{2}}{1-k r^{2}}+r^{2}\left(d \theta^{2}+\operatorname{sen}^{2} \theta d \phi^{2}\right)\right],
$$

donde $k$ denota la curvatura de las secciones espaciales del espacio-tiempo. Los posibles valores que puede tomar $k$ son $+1,0,-1$. Si $k=1$, se tiene un universo cerrado, $k=0$ corresponde a un universo plano, y $k=-1$, a un universo abierto. En ecuación (2) $a(t)$ es el factor de escala.

Las ecuaciones de Friedmann en un universo plano tipo FRW tienen la siguiente forma

$$
\begin{gathered}
H^{2}=\frac{1}{3}\left(\rho_{m}+\rho_{\Lambda}\right), \\
\dot{H}+H^{2}=-\frac{1}{6}\left(\rho_{m}+\rho_{\Lambda}+3 p_{\Lambda}\right),
\end{gathered}
$$

donde hemos considerado que $8 \pi G=1$ y la presión de la materia oscura $p_{m}=0$, ya que es un fluido tipo "polvo". $\rho_{m}$ es la densidad de materia oscura, $\rho_{\Lambda}$ es la densidad de energía oscura y $p_{\Lambda}$ es la presión debida a la componente de energía oscura. $H=\dot{a} / a$ es el parámetro de Hubble, y el punto representa derivación con respecto al tiempo. Vamos a considerar que la componente de energía oscura es la densidad de energía que tiene la siguiente forma [20]

$$
\rho_{\Lambda}=3\left(\alpha H^{2}+\beta \dot{H}\right)
$$

donde $\alpha$ y $\beta$ son constantes positivas.

Cuando se tiene en cuenta una posible interacción entre las componentes de materia oscura y de energía oscura, las ecuaciones de continuidad toman la forma

$$
\begin{gathered}
\dot{\rho}_{m}+3 H \rho_{m}=Q, \\
\dot{\rho}_{\Lambda}+3 H\left(\rho_{\Lambda}+p_{\Lambda}\right)=-Q .
\end{gathered}
$$

En este trabajo vamos a considerar el término de interacción con la siguiente forma no lineal

$$
Q=3 H b \frac{\rho_{m}^{2}}{\rho_{m}+\rho_{\Lambda}},
$$

donde $b$ es la constante de interacción. Esta forma del término de interacción es un caso particular del caso general considerado en la referencia [23], y con el cual podemos obtener soluciones analíticas y que son fenomenológicamente viables. Además, esta escogencia no ha sido considerada aún en la literatura en el escenario aquí propuesto.
Reemplazando $Q$ en la ecuación (6), usando $\rho_{m}$ de la ecuación (3) y la densidad de energía oscura, ecuación (5), se obtiene la ecuación diferencial de segundo orden no lineal para $H^{2}(x)$

$$
\begin{aligned}
& 3\left[2(\alpha-1) H^{2}(x)+\beta \frac{d}{d x} H^{2}(x)\right] \\
& {\left[2[1+b(\alpha-1)] H^{2}(x)+b \beta \frac{d}{d x} H^{2}(x)\right]} \\
& +2 H^{2}(x)\left[2(\alpha-1) \frac{d}{d x} H^{2}(x)+\beta \frac{d^{2}}{d x^{2}} H^{2}(x)\right]=0,
\end{aligned}
$$

donde se ha realizado el cambio de variable $x=\ln a$. La solución de esta ecuación diferencial es

$$
\begin{aligned}
& H^{2}(x)=A \exp \left[\frac{-2 x[3(1-b) \beta+\alpha(2+3 b \beta)]}{\beta(2+3 b \beta)}\right] * \\
& \left\{\beta e^{\left[\left(3+\frac{2}{\beta}\right) x+4 B \alpha\right]}+(2+3 b \beta) e^{\left[\frac{2 \alpha}{\beta} x+2 B(2+3 \beta)\right]}\right\}^{\frac{2}{2+3 b \beta}},
\end{aligned}
$$

donde $A$ y $B$ son constantes de integración, que se obtienen con las condiciones iniciales

$$
\begin{gathered}
H^{2}(0)=H_{0}^{2}, \\
\left.\frac{d}{d x} H^{2}(x)\right|_{x=0}=\frac{2\left(\Omega_{\Lambda 0}-\alpha\right) H_{0}^{2}}{\beta},
\end{gathered}
$$

donde $H_{0}$ y $\Omega_{\Lambda 0}$ son, respectivamente, los parámetros de Hubble y de densidad de energía oscura en el presente $(x=0, a=1)$. La condición inicial ecuación (12) se obtiene a partir de la expresión de la densidad de energía oscura dada en ecuación (5). Utilizando ecuación (11) y ecuación (12), obtenemos

$$
A=H_{0}^{2}\left[\frac{\left(\Omega_{\Lambda 0}-1\right)\left[\frac{2 \alpha-3 \beta\left[1+b\left(\Omega_{\Lambda 0}-1\right)\right]-2 \Omega_{\Lambda 0}}{\beta\left(\Omega_{\Lambda 0}-1\right)}\right]^{\frac{2+2 \beta}{2-2 \alpha+3 \beta}}}{2 \alpha-3 \beta-2}\right]^{\frac{2}{2+3 b \beta}},
$$

$$
B=\frac{\ln \left[\frac{2\left(\alpha-\Omega_{\Lambda 0}\right)-3 \beta\left[1+b\left(\Omega_{\Lambda 0}-1\right)\right]}{\beta\left(\Omega_{\Lambda 0}-1\right)}\right]}{2(2 \alpha-3 \beta-2)} .
$$

Para los cálculos numéricos vamos a utilizar los valores para $\alpha$ y $\beta$ usados en [20], es decir, $\alpha=$ 0,93 y $\beta=0,5$, con los cuales se obtiene un buen comportamiento de los parámetros $q$ y $w$. Para el parámetro de densidad de energía oscura vamos a usar el valor de uso común en la literatura, es decir, $\Omega_{\Lambda 0}=0,73$. Por lo tanto, el parámetro de interacción $b$ es el único que queda libre. 


\section{Evolución cosmológica}

Para analizar la evolución cosmológica del universo a partir del modelo anterior y poder determinar si es posible en este escenario obtener un régimen de expansión acelerada del universo en tiempos tardíos, vamos a considerar una ecuación barotrópica de estado para la densidad de energía y densidad de presión, es decir, el parámetro de la ecuación de estado viene dado por $w_{\Lambda}=p_{\Lambda} / \rho_{\Lambda}$, donde $\rho_{\Lambda}$ es

$$
\rho_{\Lambda}(x)=3\left[\alpha H^{2}(x)+\frac{1}{2} \beta \frac{d}{d x} H^{2}(x)\right] .
$$

y a partir de la ecuación (7) la densidad de presión $p_{\Lambda}$ es

$$
p_{\Lambda}(x)=-b \frac{\rho_{m}^{2}}{\rho_{m}+\rho_{\Lambda}}-\rho_{\Lambda}-\frac{1}{3} \frac{d \rho_{\Lambda}}{d x} .
$$

la expresión para la densidad de materia oscura $\rho_{m}$ se obtiene a partir de la de Friedmann ecuación (3) junto con la expresión para $\rho_{\Lambda}$ dada por la ecuación (5), es decir

$$
\rho_{m}(x)=3\left[(1-\alpha) H^{2}(x)-\frac{1}{2} \beta \frac{d}{d x} H^{2}(x)\right] .
$$

Por lo tanto, usando la ecuación para $H^{2}(x)$ dada por la ecuación (10) en las expresiones anteriores y realizando el cambio de variable $x=-\ln (1+z)$, obtenemos una expresión explícita para $w_{\Lambda}$ en términos del corrimiento al rojo $z$

$$
\begin{aligned}
& w_{\Lambda}(z)=\left\{( 2 - 2 \alpha + 3 \beta ) \left[(1+z)^{\frac{2 \alpha}{\beta}}(2 \alpha\right.\right. \\
& \left.-3 \beta\left(1+b\left(\Omega_{\Lambda 0}-1\right)\right)-2 \Omega_{\Lambda 0}\right)+3 b \beta(1+z)^{\frac{2+3 \beta}{\beta}} \\
& \left.\left.\left(\Omega_{\Lambda 0}-1\right)\right]\right\} \div\left\{3 \beta \left[-(1+z)^{\frac{2+3 \beta}{\beta}}(2 \alpha+3 \beta(b-1))\right.\right. \\
& \left(\Omega_{\Lambda 0}-1\right)+(1+z)^{\frac{2 \alpha}{\beta}}\left(-2 \alpha+3 \beta\left(1+b\left(\Omega_{\Lambda 0}-1\right)\right.\right. \\
& \left.\left.\left.+2 \Omega_{\Lambda 0}\right)\right]\right\} .
\end{aligned}
$$

En la figura 1 podemos apreciar su comportamiento. Se han considerado los valores para $b$ como $0,0,05$ y 0,1, los cuales corresponden al orden de magnitud comúnmente usado en la literatura (ver por ejemplo [34]). Estos valores serán utilizados en todos los cálculos numéricos que siguen.

Como se puede ver en la figura 1, el universo presenta una fase de expansión acelerada en tiempos tardíos, ya que se satisface la condición $w_{\Lambda}<-1 / 3$. También vemos que el parámetro de la ecuación de

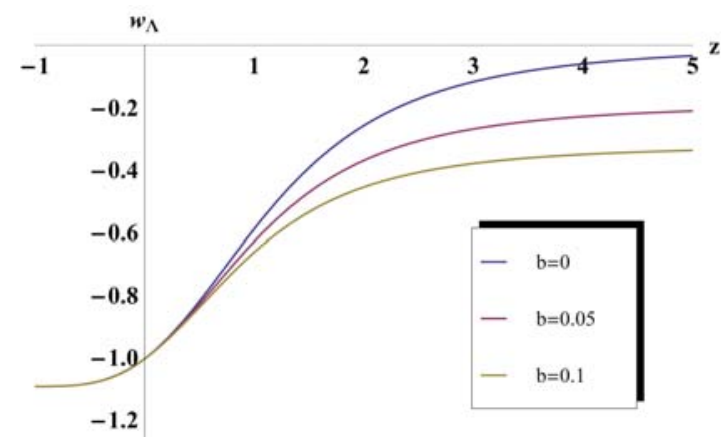

Figura 1. Parámetro de la ecuación de estado $w_{\Lambda}$ contra el corrimiento al rojo $z$.

estado cruza la barrera de -1 . En este caso el universo evoluciona de una forma que es característica de un tipo de campo escalar llamado fantasma [14]. Este tipo de comportamiento, donde la ecuación de estado toma valores mayores que -1 y también menores que -14 es característico de un tipo de modelo llamado quintom [29]. Se puede ver en la figura que el término de interacción es más apreciable en el pasado que en tiempos cercanos al presente $(z=0)$ y en el futuro $(z<0)$.

Para analizar el comportamiento de la densidad de energía oscura $\rho_{\Lambda}$ en función del corrimiento al rojo $z$ consideramos la ecuación (15). La expresión análitica para $\rho_{\Lambda}(z)$ es muy extensa para colocar aquí. En la figura 2 se puede apreciar su comportamiento

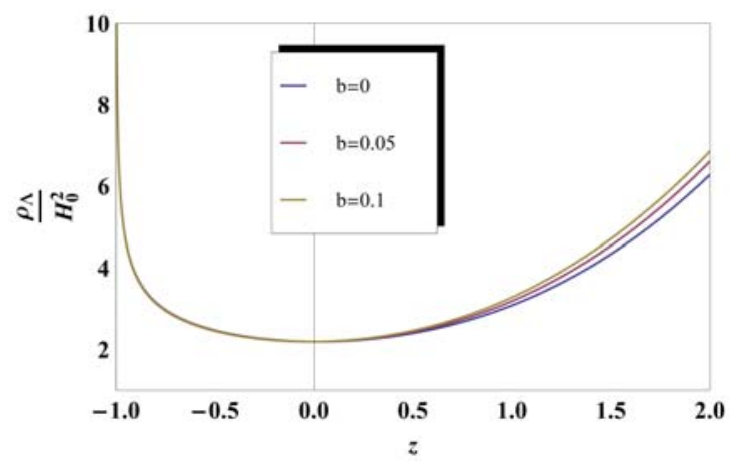

Figura 2. Densidad de energía oscura $\rho_{\Lambda}$ en función del corrimiento al rojo $z$.

La evolución de la densidad de energía oscura con respecto al corrimiento al rojo $z$ muestra que existe una singularidad tipo "Big Rip" en el futuro, la cual se mantiene para los diferentes valores de $b$ adoptados. Este tipo de singularidades son comunes en los modelos cuya ecuación de estado $w_{\Lambda}$ cruza la barrera de -1 (comportamiento tipo campo fantasma) [31]. 
Otra cantidad importante en el análisis cosmológico es el parámetro de desaceleración $q$, este parámetro se define en términos del parámetro de Hubble como

$$
q=-1-\frac{\dot{H}}{H^{2}}=-1-\frac{\left(H^{2}(x)\right)^{\prime}}{2 H^{2}(x)},
$$

donde $\left(H^{2}\right)^{\prime}=d H^{2} / d x$. Usando la ecuación (10), se tiene

$$
\begin{aligned}
q(z)= & {\left[2 \alpha-3 \beta\left(1+b\left(\Omega_{\Lambda 0}-1\right)\right)-2 \Omega_{\Lambda 0}\right] \times } \\
& {\left[(1+z)^{\frac{2 \alpha}{\beta}}(\alpha-\beta-1)\right.} \\
& \left.+\frac{(1+z)^{\frac{2+3 \beta}{\beta}} \beta[3 b(1-\alpha+\beta)-1]\left(\Omega_{\Lambda 0}-1\right)}{2 \Omega_{\Lambda 0}-2 \alpha+3 \beta\left[1+b\left(\Omega_{\Lambda 0}-1\right)\right]}\right] \\
& \div\left[( 1 + z ) ^ { \frac { 2 \alpha } { \beta } } \beta \left(2 \alpha-3 \beta\left(1+b\left(\Omega_{\Lambda 0}-1\right)\right)\right.\right. \\
& \left.\left.-2 \Omega_{\Lambda 0}\right)+(1+z)^{\frac{2+3 \beta}{\beta}} \beta(2+3 b \beta)\left(\Omega_{\Lambda 0}-1\right)\right] .
\end{aligned}
$$

Su evolución se muestra en figura 3

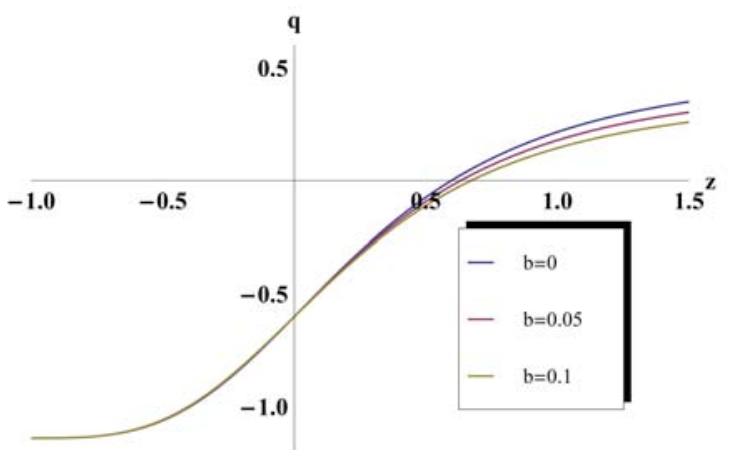

Figura 3. Parámetro de desaceleración $q$ en función del corrimiento al rojo $z$.

como se puede apreciar en la figura 3, para el parámetro de desaceleración los valores de $b$ considerados aquí generan valores del corrimiento al rojo de transición $z_{t}$ entre 0,6 y 0,7 , aproximadamente, lo cual es consistente con el valor observado $\left(z_{t} \approx 0,67\right)$ [14]. Hay que recordar que los valores positivos de $q$ corresponden a un régimen de expansión desacelerada (dominancia de materia), y los negativos, a un régimen de expansión acelerada (dominancia de la energía oscura), y la transición de un regimen al otro se da en $z_{t}$.

Finalmente, a partir de la expresión de $H^{2}(x)$ dada en la ecuación (10), podemos graficar $H(z)$ junto con los 29 valores disponibles actualmente en la literatura (ver la tabla 1 de la referencia [32]). El valor de $H_{0}$ usado aquí es el reportado por Planck 2015 [33] $\left(H_{0}=67,8 \mathrm{Km} \mathrm{s}^{-1} \mathrm{Mpc}^{-1}\right)$.

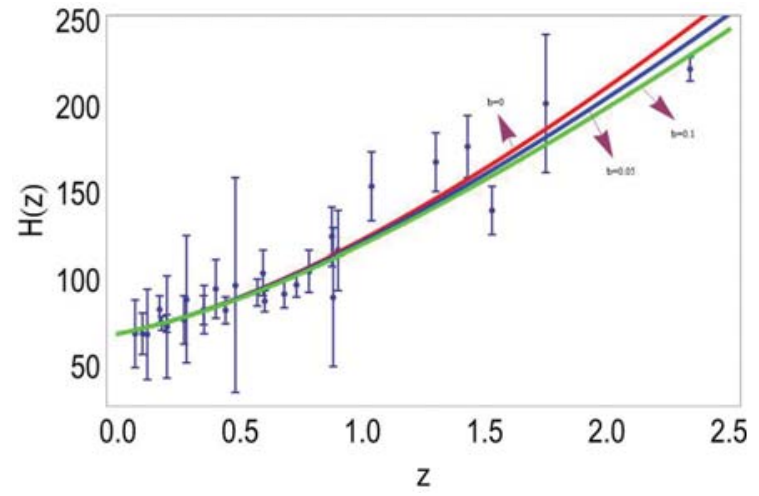

Figura 4. Parámetro de Hubble $H$ en función del corrimiento al rojo $z$. Las unidades de $H(z)$ son $\mathrm{Kms}^{-1} \mathrm{Mpc^{-1 }}$.

En la figura 4 podemos ver que el modelo cosmológico considerado en este trabajo presenta de manera cualitativa un buen "ajuste" con los datos observacionales de $H(z)$. Usualmente, la manera rigurosa de ajustar un modelo cosmológico dado con los datos observacionales (SNIa, CMB, BAO, $H(z)$ ) es mediante el uso de la técnica estadística llamada $\chi^{2}$, que en el contexto cosmológico se realiza usualmente con el programa de computadora CosmoMC, el cual utiliza el método "Markok chain Monte-Carlo" para el ajuste de los parámetros del modelo en cuestión. En este artículo no vamos a realizar este estudio detallado, el cual se podría hacer en un trabajo posterior.

\section{Conclusiones}

En este trabajo hemos considerado el modelo de energía oscura introducido en [20], teniendo en cuenta una interacción de tipo no lineal entre las componentes de energía oscura y materia oscura, dada por la ecuación (8). En este escenario obtuvimos el parámetro de la ecuación de estado correspondiente a la energía oscura $w_{\Lambda}$, el cual presenta un comportamiento tipo quintom, es decir, toma valores por encima y por debajo de la barrera de -1 (ver figura 1), y que corresponden a un régimen de expansión acelerada $\left(w_{\Lambda}<-1 / 3\right)$. La densidad de energía oscura $\rho_{\Lambda}$ presenta una singularidad en el futuro $(z \rightarrow-1)$ tipo "Big Rip", la cual es usual en los modelos tipo fantasma [31] y se mantiene para los diferentes valores del parámetro de interacción $b$ (ver figura 2). Otra de las cantidades tomadas en cuenta en este trabajo fue el parámetro de desaceleración $q$, que presenta en este contexto valores del corri- 
miento al rojo de transición $z_{t}$ que son consistentes con las observaciones $\left(z_{t} \approx 0,67\right)$ [14] (ver figura 3 ). Finalmente, analizamos el ajuste cualitativo del modelo con los datos de $H(z)$ reportados en la literatura (ver figura 4). A partir de todo lo anterior, podemos decir que el modelo de energía oscura considerado aquí junto con el término de interacción no lineal es fenomenológicamente viable.

\section{Referencias}

[1] A. G. Riess et al, "Observational evidence from supernovae for an accelerating universe and a cosmological constant", Astron. J, vol. 116, pp. 1009-1038, 1998.

[2] A. G. Riess et al, "BVRI Light curves for 22 type Ia supernovae", Astron. J, vol. 117, pp. 707-724, 1999.

[3] S. Perlmutter et al, "Discovery of a supernova explosion at half the age of the universe and its cosmological implications", Nature, vol. 391, pp. 51-54, 1998.

[4] S. Perlmutter et al, [Supernova Cosmology Project Collaboration], "Measurements of $\omega$ and $\Lambda$ from 42 high-redshift supernovae' ', Astrophys. J., vol. 517, pp. 565-586, 1999.

[5] P. Astier et al, "The supernova legacy survey: measurement of $\Omega_{M}, \Omega_{\Lambda}$ and $w$ from the first year data set", Astron. Astrophys. J., vol. 447, pp. 31-48, 2006.

[6] D. N. Spergel et al, "First year wilkinson microwave anisotropy probe (WMAP) observations: determination of cosmological parameters", Astrophys. J. Suppl., vol. 148, pp. 175-194, 2003,

[7] D. N. Spergel et al, "Wilkinson microwave anisotropy probe (WMAP) three year results: implications for cosmology", Astrophys. J. Suppl., vol. 170, pp. 377, 2007.

[8] M. Tegmark et al, "Cosmological parameters from SDSS and WMAP", Phys. Rev. D, vol. 69, pp. 103501, 2004.

[9] K. Abazajian et al, "The second data release of the sloan digital sky survey ", Astron. J., vol. 128, pp. 502-512, 2004.

[10] K. Abazajian et al, "The third data release of the sloan digital sky survey", Astron. J., vol. 129, pp. 1755-1759, 2005.
[11] S. Weinberg, "The cosmological constant problem”, Rev. Mod. Phys., vol. 61, pp. 1-23, 1989.

[12] T. Padmanabhan, "Cosmological constant - the weight of the vacuum", Phys. Rept., vol. 380, pp. 235-320, 2003.

[13] V. Sahni and A. Starobinsky, "The case for a positive cosmological Lambda-term", Int. J. Mod. Phys. D, vol. 9, pp. 373-444, 2000.

[14] E. J. Copeland, M. Sami, and Sh. Tsujikawa, "Dynamics of dark energy", Int. J. Mod. Phys. D, vol. 15, pp. 1753-1936, 2006.

[15] C. Deffayet, G. R. Dvali, and G. Gabadadze, "Accelerated universe from gravity leaking to extra dimensions", Phys. Rev. D, vol. 65, pp. 044023, 2002.

[16] S. Nojiri, and S. D. Odintsov, "Unified cosmic history in modified gravity: from $\mathrm{F}(\mathrm{R})$ theory to Lorentz non-invariant models", Phys. Rept., vol. 505, pp. 59-144, 2011.

[17] A. Y. Kamenshchik, U. Moschella and V. Pasquier, "An alternative to quintessence", Phys. Lett. B, vol. 511, pp. 265-268, 2001.

[18] S. Nojiri, and S. D. Odintsov, "Inhomogeneous equation of state of the universe: Phantom era, future singularity and crossing the phantom barrier", Phys. Rev. D, vol. 72, pp. 023003 , 2005.

[19] A. Oliveros, "Principio holográfico y su aplicación en modelos de campos escalares de energía oscura", Tesis doctoral, Departamento de Física, Universidad del Valle, Cali, 2009.

[20] L. N. Granda, and A. Oliveros, "Infrared cutoff proposal for the holographic density", Phys. Lett. B, vol. 669, pp. 275-277, 2008.

[21] V. Salvatelli, N. Said, M. Bruni, A. Melchiorri and D. Wands, "Indications of a late-time interaction in the dark sector", Phys. Rev. Lett., vol. 113, pp. 181301, 2014.

[22] Y. L. Bolotin, A. Kostenko, O. A. Lemets, and D.A. Yerokhin, "Cosmological evolution with interaction between dark energy and dark matter", Int. J. Mod. Phys. D, vol. 24, pp. 1530007, 2014.

[23] F. Arévalo, A. P. Bacalhau, and W. Zimdahl, "Cosmological dynamics with non-linear interactions", Class. Quant. Grav., vol. 29, pp. 235001, 2012. 
[24] G. Mangano, G. Miele, and V. Pettorino, "Coupled quintessence and the coincidence problem", Mod. Phys. Lett. A, vol. 18, pp. 831-842, 2003.

[25] M. Baldi, "Time dependent couplings in the dark sector: from background evolution to nonlinear structure formation", Mon. Not. Roy. Astron. Soc., vol. 411, pp. 1077, 2011.

[26] Y-Z. Ma, Y. Gong, and X. Chen, "Couplings between holographic dark energy and dark matter", Eur. Phys. J. C, vol. 69, pp. 509-519, 2010.

[27] Z. Zhang, S. Li, X-D. Li, X. Zhang, and M. Li, "Revisit of the Interaction between Holographic Dark Energy and Dark Matter", JCAP, vol. 1206, pp. 009, 2012.

[28] A. Oliveros, and M. A. Acero, "New holographic dark energy model with non-linear interaction", Astrophys. Space Sci., vol. 357, No 1, pp. 12, 2015.

[29] B. Feng, X. L. Wang, and X. M. Zhang, "Dark energy constraints from the cosmic age and supernova" Phys. Lett. B, vol. 607, pp. 35-41, 2005

[30] B. Feng, M. Li, Y. S. Piao, and X. M. Zhang, "Oscillating Quintom and the Recurrent Universe", Phys. Lett. B, vol. 634, pp. 101-105, 2006.

[31] Y-Z. Ma, "Variable cosmological constant model: its brief review, the reconstruction equation and constraints from supernova data", Nucl. Phys. B, vol. 804, pp. 262-285, 2008.

[32] X. Ding, M. Biesiada, S. Cao, Z. Li, and Z-H. Zhu, "Is there evidence for dark energy evolution?", Astrophys. J., vol. 803, pp. 2, 2015.

[33] Planck Collaboration, "Planck 2015 results. XIII. Cosmological parameters", arXiv:1502.01589 [astro-ph.CO], pp. 1-67, 2015.

[34] M. Sharif, and A. Jawad, "Cosmological evolution of interacting new holographic dark energy in non-flat universe", Eur. Phys. J. C, vol. 72, pp. 2097, 2012. 